\title{
THE MARR WAVELET PYRAMID
}

\author{
Dimitri Van De Ville, Michael Unser \\ Biomedical Imaging Group \\ École Polytechnique Fédérale de Lausanne (EPFL) \\ CH-1015 Lausanne, Switzerland
}

\begin{abstract}
We introduce a new semi-orthogonal complex wavelet basis of $L_{2}\left(\mathbb{R}^{2}\right)$. The basis functions are associated to the complex gradient-Laplace operator, which plays a central role in image processing. We define analytically a single-generator wavelet that is shifted on the coset positions of the subsampling matrix.

Next, we propose the "wavelet Marr pyramid" for an extension of the new basis that achieves near shift-invariance and steerability (using a Gaussian-like smoothing kernel), for a mild redundancy factor only. This new wavelet pyramid decomposition closely mimicks the basic operations of Marr's framework for early vision. The pyramid is implemented by a fast filterbank algorithm using the FFT.
\end{abstract}

Index Terms - complex-valued wavelets, Laplacian-ofGaussian

\section{INTRODUCTION}

The wavelet transform is a prominent tool in image processing. The 1-D transform acts as a multiscale version of an $N$ thorder derivative operator, where $N$ is the wavelet's number of vanishing moments. Multidimensional extensions are typically achieved in a separable way. However, such an approach introduces crossterms that do not have a straightforward interpretation and are not well adapted to image features such as lines and edges. The design of intrinsically 2-D wavelets has been an active field of research (wedgelets, ridgelets, contourlets, and so on).

Here, we pursue an alternative design strategy where we combine three essential ingredients of many image processing algorithms: Laplacian filtering, gradient calculation, and Gaussian smoothing. Starting from the complex gradientLaplace operator, we define associated complex polyharmonic B-splines. The spline spaces then form embedded approximation spaces, from which we can define a wavelet basis that spans the orthogonal complement. A particular feature of our approach is that the wavelets are defined from a singlegenerator wavelet (for any subsampling rate). Next, we extend the wavelet basis to a mildly-redundant pyramid structure by not subsampling the wavelet subband and exploiting the fact that a single generator wavelet is used. Moreover, we benefit from the enlarged wavelet space to further shape the wavelet function; i.e., the smoothing kernel within the wavelet is chosen to closely resemble a Gaussian.

The processing elements of the proposed decomposition closely match the ones proposed by Marr for the modeling of early vision [5]. Therefore, we coin the term "Marr wavelet pyramid".

\section{COMPLEX POLYHARMONIC B-SPLINES}

A class of differential operators of particular interest for 2-D image processing are the complex gradient-Laplace operators of the form

$$
\mathrm{L}_{\gamma, N}=(-\Delta)^{\frac{\gamma-N}{2}}\left(-j \frac{\partial}{\partial x_{1}}-\frac{\partial}{\partial x_{2}}\right)^{N}
$$

where $\gamma \in \mathbb{R}^{+}$and $N \in \mathbb{N}$. It can be shown that this is the complete family of complex operators that are simultaneously translation-invariant, scale-invariant, and rotationcovariant [1].

Complex polyharmonic B-splines associated to a complex gradient-Laplace operator are non-separable basis functions that are defined in the Fourier domain as

$$
\hat{\beta}_{\gamma, N}=\frac{V_{\gamma, N}\left(e^{j \boldsymbol{\omega}}\right)}{\hat{L}_{\gamma, N}(\boldsymbol{\omega})},
$$

where the numerator $V_{\gamma, N}\left(e^{j \boldsymbol{\omega}}\right)$ is the localization filter, and the denominator $\hat{L}_{\gamma, N}(\boldsymbol{\omega})=\|\boldsymbol{\omega}\|^{\gamma-N}\left(\omega_{1}-j \omega_{2}\right)^{N}$ is the Fourier transform of (9) in the distributional sense.

The localization filter should regularize the singularity of the Fourier transform at the origin. To that aim, we consider the polar representation $V_{\gamma, N}\left(e^{j \boldsymbol{\omega}}\right)=V_{\gamma}\left(e^{j \boldsymbol{\omega}}\right) \cdot e^{j \theta_{N}(\boldsymbol{\omega})}$ and we specify modulus and phase as

$$
\begin{aligned}
& V_{\gamma}\left(e^{j \boldsymbol{\omega}}\right)=\left(\frac{8}{3}\left(\sin ^{2}\left(\frac{\omega_{1}}{2}\right)+\sin ^{2}\left(\frac{\omega_{2}}{2}\right)\right)\right. \\
&\left.+\frac{2}{3}\left(\sin ^{2}\left(\frac{\omega_{1}+\omega_{2}}{2}\right)+\sin ^{2}\left(\frac{\omega_{1}-\omega_{2}}{2}\right)\right)\right)^{\gamma / 2}, \\
& \theta_{N}(\boldsymbol{\omega})=\angle\left(j\left[\omega_{1}\right]-j\left[\omega_{2}\right]\right)^{N},
\end{aligned}
$$


and where $[\omega]$ stands for the unique $\omega$ in $[-\pi, \pi[$ such that $\omega-[\omega]=2 n \pi$ for some integer $n$.

Notice that the choice of our localization filter is different from [2], where the phase at the origin was not compensated (i.e., $\theta_{N}(\boldsymbol{\omega})=0$ ). Our construction is also such that $\beta_{\gamma, 0}$ coincides with the isotropic polyharmonic B-splines [3] that were earlier introduced as extensions of the elementary polyharmonic B-splines [4]. Moreover, thanks to the property $\left|\hat{\beta}_{\gamma, N}\right|=\hat{\beta}_{\gamma, 0}$, the autocorrelation filter

$$
A_{\gamma}\left(e^{j \boldsymbol{\omega}}\right)=\sum_{\mathbf{k}}\left|\hat{\beta}_{\gamma}(\boldsymbol{\omega}+2 \pi \mathbf{k})\right|^{2}
$$

does not depend on $N$. Another fundamental function that is independent of $N$ is the interpolant $\phi_{2 \gamma}$ of the spline space ${ }^{1}$ associated to $\mathrm{L}_{\gamma, N}^{*} \mathrm{~L}_{\gamma, N}$, whose Fourier transform is

$$
\hat{\phi}_{2 \gamma}(\boldsymbol{\omega})=\frac{\left|\hat{\beta}_{\gamma}(\boldsymbol{\omega})\right|^{2}}{A_{\gamma}\left(e^{j \boldsymbol{\omega}}\right)} .
$$

\section{COMPLEX WAVELET BASIS}

The complex polyharmonic B-splines satisfy all the requirements for generating a multiscale analysis of $L_{2}\left(\mathbb{R}^{2}\right)$ [2]: their integer shifts form a Riesz basis; they satisfy the partition of unity property; and they satisfy a scaling relation for a subsampling matrix $\mathbf{D}$ that corresponds to a rotation combined with a dilation. In the Fourier domain, the scaling relation is given by

$$
\hat{\beta}_{\gamma, N}\left(\mathbf{D}^{T} \boldsymbol{\omega}\right)=|\operatorname{det} \mathbf{D}|^{-\frac{1}{2}} H\left(e^{j \boldsymbol{\omega}}\right) \hat{\beta}_{\gamma, N}(\boldsymbol{\omega}),
$$

where $H\left(e^{j \omega}\right)$ is the scaling filter. We consider the embedded approximation spaces

$$
\mathcal{V}_{i}=\operatorname{span}_{\mathbf{k} \in \mathbb{Z}^{2}}\left\{|\operatorname{det} \mathbf{D}|^{\frac{i}{2}} \beta_{\gamma, N}\left(\mathbf{D}^{i} \mathbf{x}-\mathbf{k}\right)\right\}
$$

to construct wavelets that span the orthogonal complements $\mathcal{W}_{i}=\mathcal{V}_{i} \ominus_{\perp} \mathcal{V}_{i-1}$. The approximation coefficients at scale $i$ are denoted as $c_{i}[\mathbf{k}]$.

\subsection{Operator-like wavelets}

One of the main difficulties in multidimensional wavelet design is the choice of the $M=|\operatorname{det} \mathbf{D}|-1$ wavelet functions that span the wavelet subspace. Here, we propose so-called "operator-like" wavelets: a single wavelet generator is shifted on $M$ positions of the cosets defined by $\mathbf{D}$. Specifically, we define the wavelets that span the detail space $\mathcal{W}_{-1}$ as

$$
\psi_{\gamma, N}^{(n)}\left(\mathbf{D}^{-1} \mathbf{x}\right)=(-\Delta)^{\frac{\gamma-N}{2}}\left(j \frac{\partial}{\partial x_{1}}-\frac{\partial}{\partial x_{2}}\right)^{N}\left\{\phi_{2 \gamma}\right\}\left(\mathbf{x}+\mathbf{e}_{n}\right),
$$$$
n=1, \ldots, M \text {, }
$$

\footnotetext{
${ }^{1}$ This "augmented order" polyharmonic space is spanned by $\beta_{\gamma, N}(\cdot) *$ $\beta_{\gamma, N}(-\cdot)=\beta_{2 \gamma, 0}$.
}

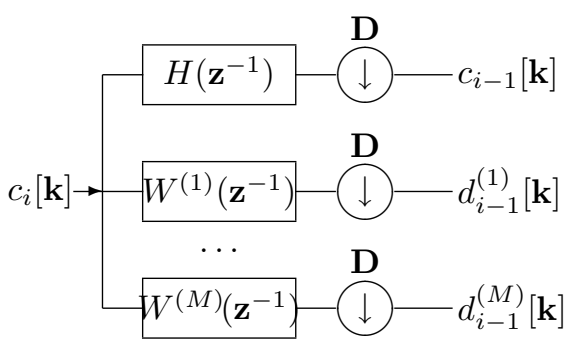

Fig. 1. Filterbank implementation of the (non-redundant) operator-like wavelet decomposition (analysis side).

where the smoothing kernel $\phi_{2 \gamma}$ is specified by (4) and $\mathbf{e}_{n}$ is in the cosets $\mathcal{C} \backslash \mathbf{e}_{0}=(0,0)$ of the subsampling matrix $\mathbf{D}$. As we write the generator wavelet as $\psi_{\gamma, N}^{(0)}=\psi_{\gamma, N}$, we can compactly denote the wavelet subspaces $\mathcal{W}_{i}$ as

$$
\mathcal{W}_{i}=\operatorname{span}\left\{|\operatorname{det}(\mathbf{D})|^{i / 2} \psi_{\gamma, N}\left(\mathbf{D}^{i} \mathbf{x}-\mathbf{D}^{-1} \mathbf{k}\right)\right\}_{\mathbf{k} \in \mathbb{Z}^{2} \backslash \mathbf{D} \mathbb{Z}^{2}}
$$

We prove elsewhere [1] that operator-like wavelets generate a semi-orthogonal basis of $L_{2}\left(\mathbb{R}^{2}\right)$.

We use a filterbank implementation to compute the wavelet decomposition. The wavelet filters can be obtained by expressing $\psi_{\gamma, N}^{(n)}$ as a function of the scaling function $\beta_{\gamma, N}$ :

$$
\hat{\psi}_{\gamma, N}^{(n)}\left(\mathbf{D}^{T} \boldsymbol{\omega}\right)=|\operatorname{det} \mathbf{D}|^{-1} \underbrace{\frac{V_{\gamma, N}^{*}\left(e^{j \boldsymbol{\omega}}\right)}{A_{\gamma}\left(e^{j \boldsymbol{\omega}}\right)} e^{j \boldsymbol{\omega}^{T} \mathbf{e}_{n}}}_{W^{(n)}\left(e^{j \boldsymbol{\omega}}\right)} \hat{\beta}_{\gamma, N}(\boldsymbol{\omega})
$$

In Fig. 1, we show the analysis side of the filterbank for one decomposition level. The filters at the synthesis side are found by determining the (unique) dual scaling function and wavelets within the same function spaces, or, equivalently, by expressing the perfect reconstruction condition. The filter operations are efficiently implemented in the Fourier domain using the same procedure as in [3]. The wavelet coefficients are complex-valued and thus bring along a redundancy factor of 2 for real-valued images.

From now on, we restrict ourselves to the dyadic subsampling scheme $\mathbf{D}=\operatorname{diag}(2,2)$.

\section{MARR WAVELET PYRAMID}

\subsection{Marr's theory of vision}

The complex gradient-Laplace operator plays an important role in many image processing tasks, but also in the modeling of the early stage of the primate's visual system, as proposed by David Marr in his seminal work on vision [5]. The basic operations in this framework are image smoothing by a Gaussian, application of the Laplacian operator, and detecting zero-crossing with their orientations. The last step can be computationally performed by means of the complex gradient 
operator; i.e., the zero crossings of the Laplacian are mapped onto local extrema of the complex gradient-Laplace operator. Additionally, the local orientation and strength of the edge can be recovered from the phase and modulus of the wavelet coefficient.

\subsection{Marr wavelet pyramid}

We now tailor our operator-like wavelet decomposition so that it best replicates the operations of Marr's theory, in a multiscale way. To that aim, we propose:

1. The choice for the parameters of the operator $\mathrm{L}_{\gamma, N}$ to correspond to the Marr preprocessing steps is $\gamma=3$, $N=1$; i.e., the pure Laplacian is combined with the complex gradient.

2. The three wavelets $\psi_{3,1}^{(n)}, n=1, \ldots, 3$, originate from the same generator function $\psi_{3,1}$. Therefore, it is tempting to rearrange them in a single subband and add the "missing" shift (for $\mathbf{e}_{0}=\left[\begin{array}{ll}0 & 0\end{array}\right]^{T}$ ). This is equivalent to not subsampling the wavelet subband after filtering with $W^{(0)}\left(e^{j \boldsymbol{\omega}}\right)$. Consequently, we obtain the enlarged wavelet spaces

$$
\mathcal{W}_{i}^{+}=\operatorname{span}\left\{\psi_{3,1}\left(2^{i} \mathbf{x}-\mathbf{k} / 2\right)\right\}_{\mathbf{k} \in \mathbb{Z}^{2}} .
$$

The (non-subsampled) wavelet coefficients at scale $i$ are denoted as $d_{i}[\mathbf{k}]$. Clearly, this extension improves the shift-invariance of the decomposition.

3. The smoothing kernel $\phi_{2 \gamma}$ of our original wavelet is an interpolant, which is not very Gaussian-like. We exploit the additional freedom from the enlarged wavelet space to build a wavelet with a smoothing kernel that better resembles the Gaussian; i.e., we filter the wavelet coefficients with $A\left(e^{-j \boldsymbol{\omega}}\right)$, which turns $\phi_{2 \gamma}$ into $\beta_{2 \gamma}$. The latter function is known to converge to a Gaussian as $\gamma$ increases [3]. The generator wavelet can now be written as

$$
\psi(\mathbf{x} / 2)=\Delta\left(j \frac{\partial}{\partial x_{1}}-\frac{\partial}{\partial x_{2}}\right)\left\{\beta_{2 \gamma}\right\}(\mathbf{x}) .
$$

Thanks to the Gaussian-like smoothing kernel, the Marr wavelet (11) becomes quasi-steerability; i.e., $\operatorname{Re}\{\psi\}$ can be rotated at any angle by a suitable combination with $\operatorname{Im}\{\psi\}$. The difference with the operator-like wavelet can be appreciated in Fig. 2.

In Fig. 3, we show the filterbank implementation of the wavelet pyramid. The redundancy factor introduced by the pyramid structure is only $4 / 3$, bringing the total redundancy to $8 / 3$, which is comparable to Simoncelli's steerable pyramid with 2 orientations [6].

Reconstruction from the pyramid decomposition is achieved using a so-called "subband regression" technique [7], which projects the redundant wavelet subband into

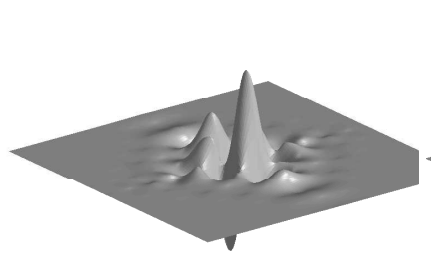

(a)

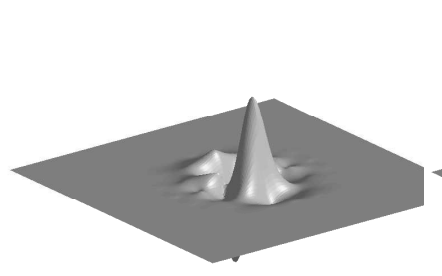

(c)

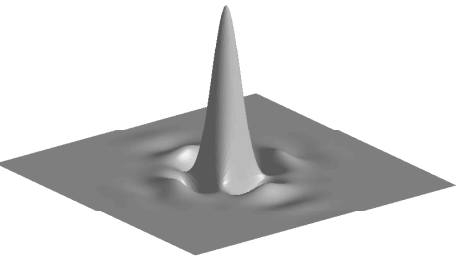

(b)

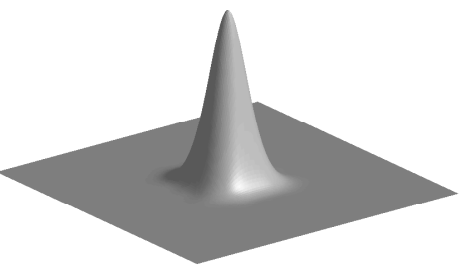

(d)

Fig. 2. (a) Operator-like wavelet of (9) with $\gamma=3, N=1$ with (b) smoothing kernel within, which is an interpolant. (c) Marr wavelet of (11) with (d) smoothing kernel within, which is Gaussian-like.

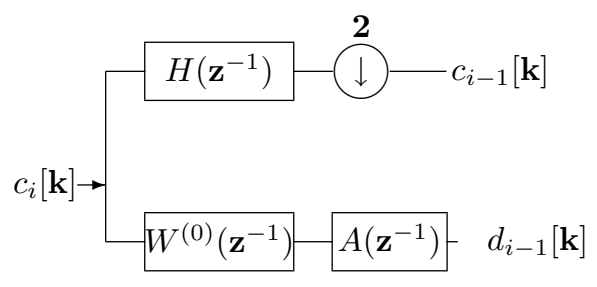

Fig. 3. Filterbank implementation of the Marr wavelet pyramid (analysis side).

the non-redundant one by optimizing a consistency measure. This scheme provides perfect reconstruction and exploits the redundancy in case of in-band processing.

\section{EXAMPLE}

In Fig. 4 (a), we show the decomposition of the test image "cameraman" for the operator-like wavelets $(\gamma=3, N=1)$. At each decomposition level, the $M=3$ wavelet subbands are arranged according to the usual scheme that is used for 2-D separable wavelet decomposition. In (b), the subbands have been rearranged and the missing shift has been added to obtain the pyramid structure; we have also tuned the wavelet by reshaping the smoothing kernel to the polyharmonic Bspline $\beta_{6}$.

We demonstrate the angular selectivity of the Marr wavelet pyramid. Reconstruction from coefficients from Fig. 4 (c) with phase inside and outside the angular wedge $\pi / 4 \pm \pi / 8$ have been selected, and are shown in Fig. 5 (a) and (b), respectively. Features with the proper orientation are separated from the image. 

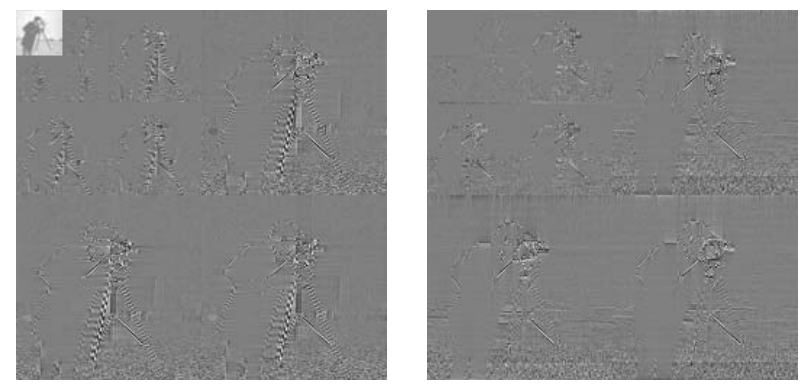

(a)

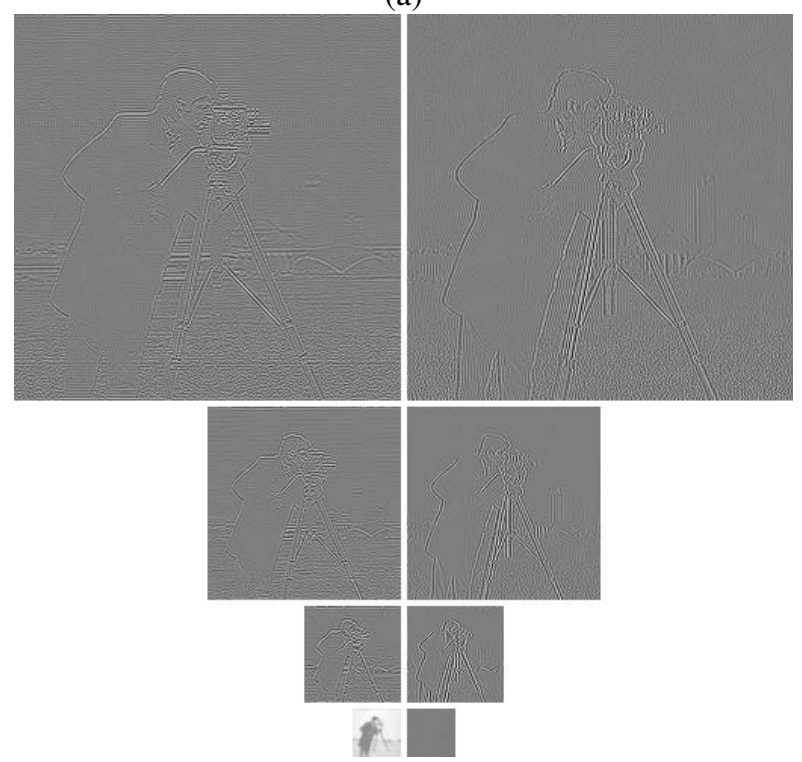

(b)

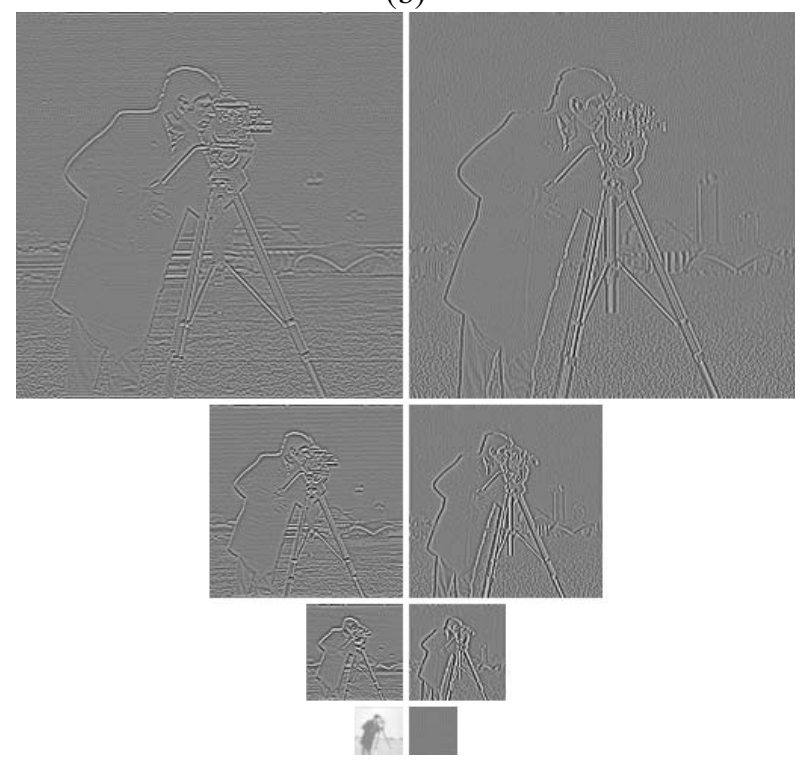

(c)

Fig. 4. Decomposition of test image "cameraman". (a) Nonredundant operator-like wavelet decomposition $(\gamma=3$, $N=1$ ). Real and imaginary part left and right, respectively. (b) Pyramid decomposition. (c) Marr wavelet pyramid.

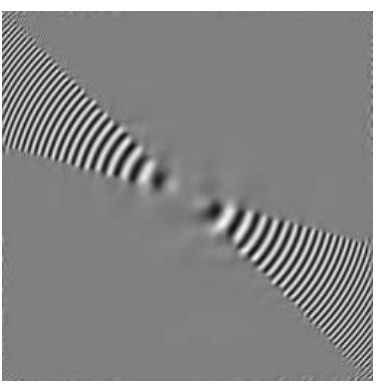

(a)

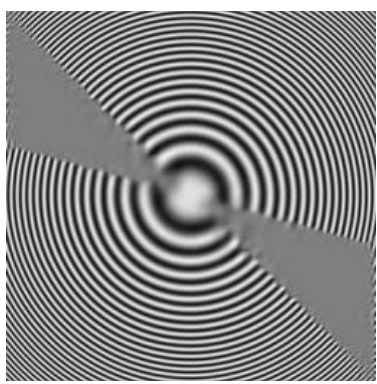

(b)
Fig. 5. Demonstration of the angular selectivity of the Marr wavelet pyramid (8 decomposition levels). Coefficients with phase inside and outside the wedge $\pi / 4 \pm \pi / 8$ are selected and reconstruction in (a) and (b), respectively.

\section{CONCLUSION \& OUTLOOK}

We proposed a new wavelet design initiated by the complex gradient-Laplace operator that plays an essential role in image processing. We obtain a complex wavelet basis, which is extended to a pyramid-type decomposition with limited redundancy (i.e., a factor $8 / 3$ ).

In future work, we will further tighten the link with Marr's theory of vision. In particular, we will extract the a compact multiscale primal sketch from the Marr pyramid. We will also investigate the problem of image reconstruction from this primal sketch representation. The Marr pyramid would provide an economical alternative for this task with respect to a fully redundant approach [8].

\section{ACKNOWLEDGEMENTS}

This was supported in part by the Swiss National Science Foundation under Grant 200020-109415 and in part by the Center for Biomedical Imaging (CIBM) of the Geneva-Lausanne Universities and the EPFL, as well as the foundations Leenaards and Louis-Jeantet.

\section{REFERENCES}

[1] D. Van De Ville and M. Unser, "Complex wavelet bases, steerability, and the Marr pyramid," submitted.

[2] B. Forster, T. Blu, D. Van De Ville, and M. Unser, "Shiftinvariant spaces from rotation-covariant functions," Applied and Computational Harmonic Analysis, in press.

[3] D. Van De Ville, T. Blu, and M. Unser, "Isotropic polyharmonic B-splines: Scaling functions and wavelets," IEEE Transactions on Image Processing, vol. 14, no. 11, pp. 1798-1813, Nov. 2005.

[4] C. Rabut, "Elementary $m$-harmonic cardinal B-splines," $\mathrm{Nu}$ merical Algorithms, vol. 2, pp. 39-62, 1992.

[5] D. Marr and E. Hildreth, "Theory of edge detection," Proceedings of the Royal Society of London. Series B, Biological Sciences, vol. 207, no. 1167, pp. 187-217, feb 1980.

[6] E. P. Simoncelli, W. T. Freeman, and E. H. a. Adelson, "Shiftable multi-scale transforms," IEEE Transactions on Information Theory, vol. 38, no. 2, pp. 587-607, mar 1992.

[7] M. Unser and D. Van De Ville, "The pairing of a wavelet basis with a mildly redundant analysis via subband regression," IEEE Transactions on Image Processing, submitted.

[8] S. Mallat and S. Zhong, "Characterization of signals from multiscale edges," IEEE Transactions on Pattern Analysis and Machine Intelligence, vol. 14, no. 7, pp. 710-732, July 1992. 